\title{
A Case of KIT-Negative Extra-Gastrointestinal Stromal Tumor of the Lesser Omentum
}

\author{
Hisataka Ogawa ${ }^{a}$ Kunihito Gotoh ${ }^{a}$ Terumasa Yamada ${ }^{a}$ \\ Hidenori Takahashi $^{\mathrm{a}}$ Hiroaki Ohigashi $^{\mathrm{a}}$ Shigenori Nagata ${ }^{\mathrm{b}}$ \\ Yasuhiko Tomita ${ }^{b}$ Masahiko Yano ${ }^{a}$ Osamu Ishikawa ${ }^{a}$ \\ Departments of ${ }^{a}$ Surgery and ${ }^{b}$ Cytology and Pathology, Osaka Medical Center \\ for Cancer and Cardiovascular Diseases, Osaka, Japan
}

\section{Key Words}

Extra-gastrointestinal stromal tumor $\cdot$ KIT-negative $\cdot$ Lesser omentum

\begin{abstract}
We report the unique case of a 69-year-old man with an extra-gastrointestinal stromal tumor (EGIST) in the lesser omentum. Based on the location of the tumor and the radiological findings, we made a provisional diagnosis of hepatic cavernous hemangioma in the lateral segment. However, after 5 years of follow-up, tumor growth was noted and the patient underwent a laparotomy. The tumor was located in the lesser omentum and resected en bloc with its fused lesser omentum and an adherent portion of the liver. The pathology results indicated an EGIST with microscopic proliferation of epithelioid cells in the lesser omentum; the tumor was immunohistochemically negative for KIT staining and positive for CD34 and PKC theta. Because of the rarity of mitotic figures and the low Ki-67 labeling, the tumor was diagnosed as a KIT-negative EGIST with a low malignant potential. The patient was followed up without receiving imatinib mesylate treatment and has remained free of any signs of recurrence for 26 months. The present case report describes a very rare lesser omental KIT-negative EGIST.
\end{abstract}

\section{Introduction}

Since Hirota et al. [1] first described a mesenchymal tumor of the stomach with a c-kit mutation, gastrointestinal stromal tumor (GIST) has become the most common mesenchymal tumor of the gastrointestinal tract. GISTs can appear anywhere in the gastrointestinal tract, although they are most commonly found in the stomach (40-70\%), small intestine (20-40\%) and colon (5-15\%) as well as the esophagus. 
GISTs are capable of differentiating along the lines of interstitial cells of Cajal, which among the cells of the gastrointestinal tract are the only ones capable of expressing KIT and CD34. KIT has been identified in virtually all GISTs. Approximately $70-80 \%$ of GISTs are also positive for CD34. Nevertheless, previous studies have indicated the existence of KIT-negative GISTs [2-4].

Recently, extra-gastrointestinal stromal tumors (EGISTs) showing the features of GIST have been described at extra-gastrointestinal sites. Omental, mesenteric and retroperitoneal EGIST are the most common sites and comprise just less than $5 \%$ of all GISTs [5]. Lesser omental EGISTs, in particular, are very rare. Only six lesser omental EGISTs have been reported to date [6-8]. All six cases were positive for KIT. In this report, we present the first case report of a lesser omental KIT-negative EGIST.

\section{Case Report}

A 69-year-old Japanese man was referred to our internal medicine department for the treatment of an abdominal mass detected during abdominal ultrasonography (AUS) performed as part of a health examination. The patient had no symptoms, and the findings of physical and laboratory tests, including hepatitis B and C virus markers, were unremarkable. The patient did not have a history of previous illness and was not taking any regular medications. The AUS demonstrated a $7 \times 4 \mathrm{~cm}$ isoechogenic mass with an internal cystic component in the lateral segment of the liver. Magnetic resonance imaging with gadolinium revealed a $7 \times 4 \mathrm{~cm}$, well-circumscribed hepatic tumor located in the lateral segment adjacent to the stomach. The mass had a high signal intensity on T2-weighted images and a characteristic enhancement pattern on postgadolinium T1-weighted images, showing peripheral, discontinuous nodular enhancement on the early phase and progressive, nearly complete central filling enhancement on delayed images. A contrast-enhanced CT (CECT) scan showed initial enhancement of the lobulated region, followed by gradual enhancement of the whole mass with some heterogeneity (fig, 1a).

At first, the mass was considered a benign tumor with hypervascularity, such as a hepatic cavernous hemangioma or focal nodular hyperplasia. The patient was followed up using an AUS examination once a year. Five years after the first examination, AUS examination revealed an enlargement of the cystic component. A CECT scan showed rapid enlargement of the lateral cystic component of the mass, suggesting an internal hemorrhage (fig. 1b). An endoscopic US examination revealed an $8 \times 4 \mathrm{~cm}$ low-echoic lesion that had not adhered to the side of the lesser omentum of the stomach, and a biopsy of the mass was impossible. Because of the enlargement of the mass, we performed a laparotomy. The mass had arisen from the lesser omentum and was slightly adherent to the lateral-ventral segment of the liver. We resected the tumor together with a partial hepatectomy of the adhered segment without perforating the capsule of the tumor. Macroscopically, a $16 \mathrm{~cm}$ tumor was found surrounded by a thin pseudocapsule of varying size but with no direct attachment to the liver (fig. 2a). When sectioned, the tumor was found to be composed of solid and cystic components. The solid component was whitish-brown in color and was an elastic, hard tumor (fig. 2b). The cystic part had a honeycomb-like appearance with clotted blood inside. Microscopically, the solid part of the tumor showed proliferation of epithelioid cells with uniformly oval to rounded nuclei and pale eosinophilic cytoplasm arranged in a sheet-like pattern ( $\mathrm{fig}$. 3a). Immunohistochemistry revealed the tumor cells to be negative for KIT (fig. 3b), desmin and S100 and positive for CD34 (fig. 3c) and PKC theta (fig. 3d). Mitotic figures were rare. The Ki-67 labeling index was below 1\%. Because of these findings, the tumor was diagnosed as a KIT-negative EGIST in the lesser omentum and was thought to have a low malignant potential. The patient was followed up without imatinib mesylate treatment and has remained free of recurrence for 26 months since the operation. 


\section{Discussion}

EGIST comprises only about 5-7\% of all GISTs. Because of the rarity of EGIST, the clinicopathological, immunohistochemical and genetic features of EGIST have not been fully elucidated. Approximately $80 \%$ of EGISTs are located in the omentum or mesentery. Lesser omental GISTs are particularly rare. Only six reports have been previously reported [6-8].

Several recent studies have shown a strong analogy between GISTs and EGISTs from a histological aspect. Histologically, EGISTs exhibit the characteristic changes seen in GISTs, with rounded epithelioid to spindle-shaped cells with a fine fibrillary background and varying amounts of myxoid and hyalinized stroma [9]. In most cases, GISTs may be identified using light microscopy. Moreover, pathologists commonly use a panel of immunohistochemical markers to confirm the morphological impression. The recommended panel is commonly comprised of CD117, anti-CD34, smooth muscle actin, desmin, and S100. CD117 is a specific marker corresponding to the KIT protein. Similar to GISTs, immunohistochemical examinations of EGISTs are typically positive for KIT and less consistently positive for CD34 and smooth muscle actin but almost always negative for desmin and S100 [5]. Moreover, the genetic pattern of c-kit and platelet-derived growth factor receptor (PDGFRA) gene mutations found in EGISTs are similar to those found in GISTs [10]. Therefore, c-kit and PDGFRA gene mutations may play important roles in the tumorigenesis of EGISTs, similar to GISTs. However, it is well known that a small number of GISTs and EGISTs do not exhibit functional mutations in c-kit and do not stain positive for KIT.

In KIT-negative cases, the diagnosis of GIST can be difficult. From a clinical perspective, an accurate diagnosis is essential in view of the availability of specific, pathogenesis-targeted treatments involving the c-kit/PDGFRA tyrosine kinase inhibitor imatinib mesylate and newer agents as targeted oncologic therapy. Some populations of KIT-negative GISTs may benefit from imatinib treatment. Neoplasms possessing a morphology compatible with GIST but exhibiting weak or no staining for KIT should undergo molecular diagnostic testing for gain-of-function mutations in the c-kit and PDGFRA genes. However, many hospitals cannot readily perform molecular diagnostic testing because of its unavailability. Recent studies have shown that PKC theta is not only sensitive, but also specific as a diagnostic marker for GIST. PKC is a serine/threonine kinase that controls cell proliferation, differentiation and motility and is expressed in $72-100 \%$ of GISTs, as shown using immunohistochemistry $[2,11]$. Though additional studies on larger populations are needed to confirm the usefulness of PKC theta, because of the small number of KIT-negative GISTs, PKC theta may be a useful marker for the diagnosis of neoplasms possessing a morphology compatible with GIST.

In the present case, based on the morphology and immunohistochemical results including tests for KIT and PKC theta, we were able to diagnose the tumor accurately as a KIT-negative EGIST developing in the lesser omentum. The current definitive treatment of GIST, including EGIST, is a complete surgical en bloc resection. An extensive lymphadenectomy is not required because of the low incidence $(<10 \%)$ of lymph node metastasis. GIST is thought to be a potentially malignant tumor, and the malignant potential has been assessed using various parameters, such as tumor size, mitotic figures, necrosis, cell type and Ki-67 labeling [12,13]. Because of the rarity of 
EGIST, the malignant potential of EGISTs must be predicted based on prognostic factors for GIST. Most EGISTs are located in the mesentery, omentum and retroperitoneum, where the tumors may not cause any symptoms until they are very large; thus, the size criterion is not as important for the prognosis of EGIST as it is for that of GIST [10]. In fact, in seven previously reported cases, tumor diameters ranged from 2.7 to $17 \mathrm{~cm}$ (median $11.5 \mathrm{~cm}$ ). A recent report showed that the prognosis of EGIST can be predicted based on a combination of the mitotic rate and the Ki-67 labeling index. In the study by Yamamoto et al. [10], a high mitotic rate ( $>5 / 50 \mathrm{HPF})$ and a high Ki-67 labeling index $(>10 \%)$ were each significantly associated with an adverse outcome. In our case, mitotic figures were rare, and the Ki-67 labeling index was $<1 \%$. These data suggested that our case has a low risk of malignancy. We achieved an R0 resection with a sufficient surgical margin. The patient was followed up without imatinib mesylate treatment. However, careful follow-up will continue to be needed in the future because of the high recurrence rate [14].

In summary, EGISTs are rare, but should be considered when investigating mesenchymal tumors located outside the gastrointestinal tract. Complete resection of the tumor is the treatment of choice. The recognition of microscopic patterns and characteristic immunohistochemical phenotypes, including KIT and CD34, is mandatory for obtaining an accurate diagnosis. However, small populations of EGIST are negative for KIT. PKC theta may be another useful marker for KIT-negative EGISTs.
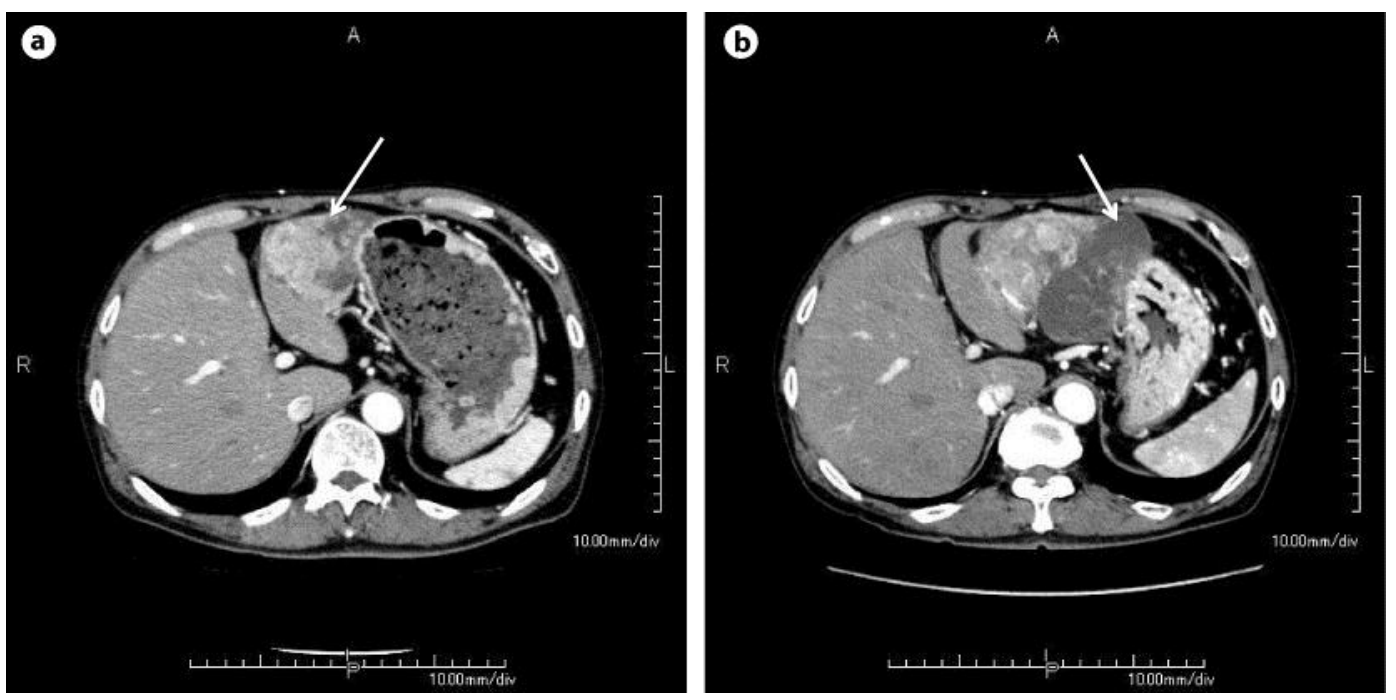

Fig. 1. a CECT shows a $7 \times 4 \mathrm{~cm}$ hypervascular tumor with heterogeneity located in the lesser omentum (arrow). b CECT shows a lateral cystic component of the tumor enlarged with an internal hemorrhage (arrow). 


\begin{tabular}{r|l|l|l}
$\begin{array}{r}\text { Case Reports in } \\
\text { Gastroenterology }\end{array}$ & $\begin{array}{l}\text { Case Rep Gastroenterol 2012;6:375-380 } \\
\text { DOI: 10.1159/000337908 }\end{array}$ & $\begin{array}{l}\text { Published online: } \\
\text { June 19, 2012 }\end{array}$ & $\begin{array}{l}\text { @ 2012 S. Karger AG, Basel } \\
\text { ISSN 1662-0631 } \\
\text { www.karger.com/crg }\end{array}$ \\
\hline
\end{tabular}
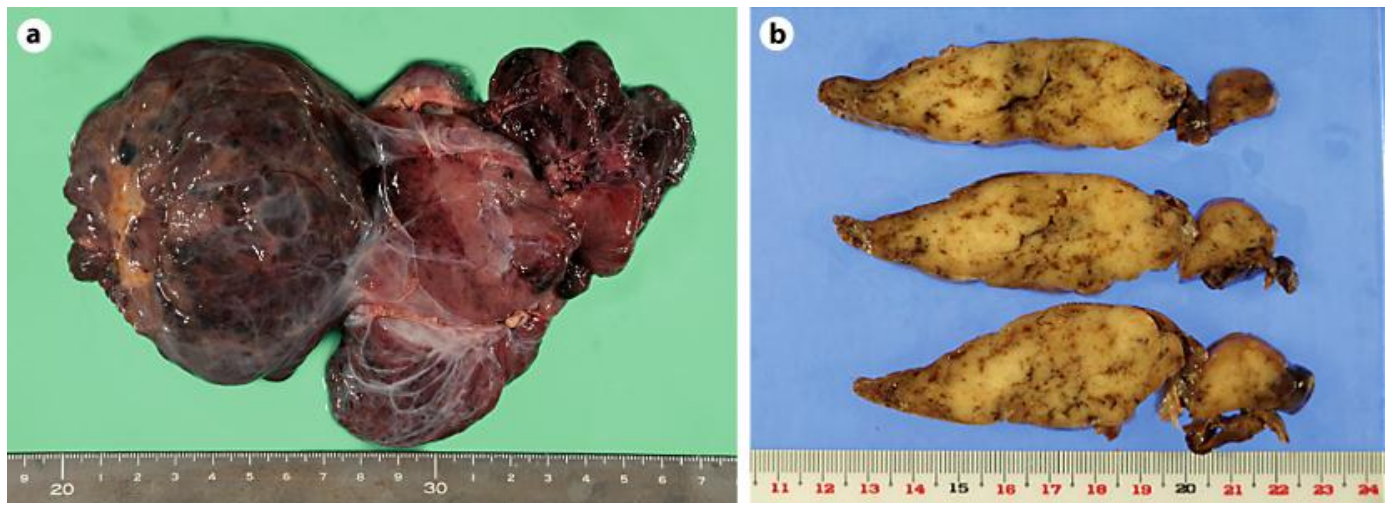

Fig. 2. a The resected tumor was a $16 \mathrm{~cm}$ solid tumor surrounded by a thin pseudocapsule of varying size. $\mathbf{b}$ When the tumor was sectioned, the solid component appeared as a whitish-brown, elastically hard mass.
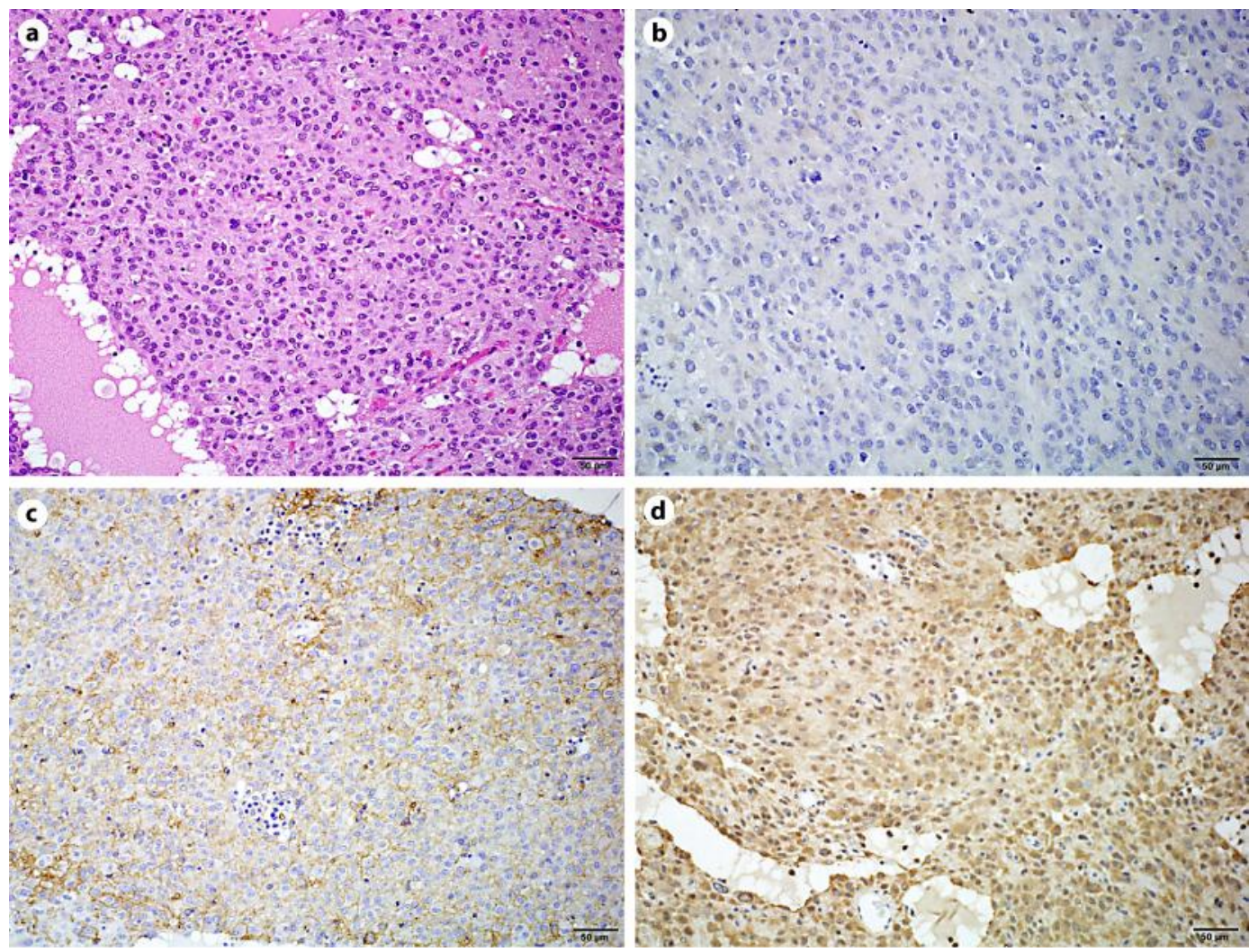

Fig. 3. a Microscopic findings show the proliferation of epithelioid cells with uniformly oval to

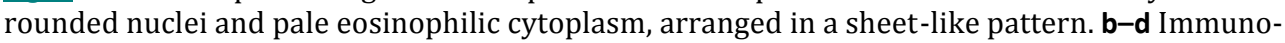
histochemical staining showed the tumor to be negative for KIT (b) and positive for CD34 (c) and PKC theta (d). 


\section{References}

1 Hirota S, Isozaki K, Moriyama Y: Gain-of-function mutations of c-kit in human gastrointestinal stromal tumors. Science 1998;279:577-580.

$\checkmark 2$ Lee HE, Kim MA, Lee HS, Lee BL, Kim WH: Characteristics of KIT-negative gastrointestinal stromal tumours and diagnostic utility of protein kinase C theta immunostaining. J Clin Pathol 2008;61:722-729.

3 Sevinc A, Camci C, Yilmaz M, Buyukhatipoglu H: The diagnosis of C-kit negative GIST by PDGFRA staining: clinical, pathological, and nuclear medicine perspective. Onkologie 2007;30:645-648.

4 Medeiros F, Corless CL, Duensing A: KIT-negative gastrointestinal stromal tumors: proof of concept and therapeutic implications. Am J Surg Pathol 2004;28:889-894.

5 Miettinen M, Monihan JM, Sarlomo-Rikala M: Gastrointestinal stromal tumors/smooth muscle tumors (GISTs) primary in the omentum and mesentery: clinicopathologic and immunohistochemical study of 26 cases. Am J Surg Pathol 1999;23:1109-1118.

-6 Nakaya I, Iwata Y, Abe T, Yokoyama H, Oda Y, Nomura G: Malignant gastrointestinal stromal tumor originating in the lesser omentum, complicated by rapidly progressive glomerulonephritis and gastric carcinoma. Intern Med 2004;43:102-105.

-7 Aihara R, Ohno T, Mochiki E: Gastrointestinal stromal tumor of the lesser omentum in a young adult patient with a history of hepatoblastoma: report of a case. Surg Today 2009;39:349-352

-8 Sakurai S, Hishima T, Takazawa Y: Gastrointestinal stromal tumors and KIT-positive mesenchymal cells in the omentum. Pathol Int 2001;51:524-531.

$>9$ Reith JD, Goldblum JR, Lyles RH, Weiss SW: Extragastrointestinal (soft tissue) stromal tumors: an analysis of 48 cases with emphasis on histologic predictors of outcome. Mod Pathol 2000;13:577-585.

10 Yamamoto H, Oda Y, Kawaguchi K: c-kit and PDGFRA mutations in extragastrointestinal stromal tumor (gastrointestinal stromal tumor of the soft tissue). Am J Surg Pathol 2004;28:479-488.

11 Motegi A, Sakurai S, Nakayama H, Sano T, Oyama T, Nakajima T: PKC theta, a novel immunohistochemical marker for gastrointestinal stromal tumors (GIST), especially useful for identifying KIT-negative tumors. Pathol Int 2005;55:106-112.

12 Miettinen M, Lasota J, Sobin LH: Gastrointestinal stromal tumors of the stomach in children and young adults: a clinicopathologic, immunohistochemical, and molecular genetic study of 44 cases with long-term follow-up and review of the literature. Am J Surg Pathol 2005;29:1373-1381.

13 Singer S, Rubin BP, Lux ML: Prognostic value of KIT mutation type, mitotic activity, and histologic subtype in gastrointestinal stromal tumors. J Clin Oncol 2002;20:3898-3905.

14 Pidhorecky I, Cheney RT, Kraybill WG, Gibbs JF: Gastrointestinal stromal tumors: current diagnosis, biologic behavior, and management. Ann Surg Oncol 2000;7:705-712. 